\title{
Activation of mitogen-activated protein kinases during preparation of vein grafts and modulation by a synthetic inhibitor
}

\author{
Costas Bizekis, MD \\ Giuseppe Pintucci, PhD \\ Christopher C. Derivaux, MD \\ Fiorella Saponara, BA \\ Jin-Hee Kim, BS \\ Kevin M. Hyman, MD \\ Eugene A. Grossi, MD \\ F. Gregory Baumann, $\mathrm{PhD}$ \\ Paolo Mignatti, MD \\ Aubrey C. Galloway, MD
}

\begin{abstract}
From The Seymour Cohn Cardiovascular Research Laboratory, Division of Cardiothoracic Surgery, Department of Surgery, New York University School of Medicine, New York, NY.

Preliminary results presented at the October 2001 Meeting of the American College of mmol/L PD98059 (mitogen-activated protein kinase kinase-1/2 inhibitor) until the vein was grafted (mean 50 minutes). To study kinetics of intracellular signaling pathways, canine saphenous veins were harvested, and mitogenactivated protein kinases and PI-3 kinase pathways were studied after different incubation time intervals. Extracted proteins were analyzed by Western blotting or in vitro kinase assay.
\end{abstract} Surgeons.

Received for publication May 9, 2002; revisions requested Aug 22, 2002; revisions received Sept 16, 2002; accepted for publication Oct 29, 2002.

Address for reprints: Aubrey C. Galloway, MD, Professor of Surgery, Director, Cardiac Surgical Research, New York University School of Medicine, 530 First Avenue, Suite 9V, New York, NY 10016 (E-mail: galloway@cv.med.nyu.edu).

J Thorac Cardiovasc Surg 2003;126:659-65

Copyright () 2003 by The American Association for Thoracic Surgery

$0022-5223 / 2003 \$ 30.00+0$

doi:10.1016/S0022-5223(03)00075-8

Results: The human saphenous veins showed elevated levels of active extracellular signal-regulated kinase after harvesting $(\mathrm{t}=0)$ and prior to implant $(\mathrm{t}=$ 1). Incubation with PD98059 resulted in decreased activation of extracellular signal-regulated kinase. Kinetics of canine saphenous veins showed extracellular signal-regulated kinase and c-jun $\mathrm{N}$-terminal kinase activation, in a timedependent manner, along with activation of the growth factor-regulated PI3 kinase pathway.

Conclusions: This study characterizes activation of extracellular signal-regulated kinases and c-jun $\mathrm{N}$-terminal kinases during vein graft preparation and demonstrates
Objective: Long-term durability of saphenous vein grafts used for coronary artery bypass grafting is limited by neointimal formation. Arterial vascular injury is known to activate intracellular mitogen-activated protein kinases, including extracellular signal-regulated kinases and c-jun N-terminal kinases, that affect cell differentiation, proliferation, migration, and apoptosis. This study tests the hypothesis that these mitogen-activated protein kinases are activated in saphenous veins during preparation for coronary artery bypass grafting.

Methods: Saphenous veins were harvested from 10 patients undergoing coronary artery bypass grafting. A specimen from each vein was placed in ice-cold lysis buffer immediately after harvesting $(\mathrm{t}=0)$. The remaining tissue was incubated at room temperature in normal saline, $0.1 \%$ dimethylsulfoxide (vehicle), or 50 the ability to inhibit extracellular signal-regulated kinase activation by simple 
incubation with a specific inhibitor. Further studies are needed to evaluate the significance of these findings with respect to graft durability.

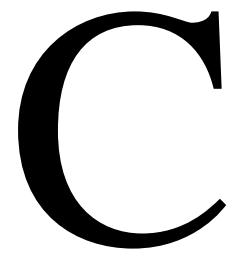

ardiovascular surgery and interventional cardiology in patients with coronary artery disease can prevent myocardial infarction and reduce morbidity and mortality. Typically, coronary revascularization is accomplished by either percutaneous transluminal coronary angioplasty, with or without the placement of endovascular stents, or by coronary artery bypass grafting, using either arterial or venous conduits.

The major impediment to long-term patency after angioplasty is restenosis; a similar limiting factor after bypass grafting is the development of intimal hyperplasia ${ }^{1-3}$ with late graft failure. Both processes have a significant late impact on the outcome of each medical intervention. Although the 10-year patency of a left internal mammary artery bypass graft approaches $95 \%$, the 5- and 10-year patency of a saphenous vein graft (SVG) is approximately $74 \%$ and $41 \%$, respectively. ${ }^{4}$ When graft failure occurs, patients usually require vascular intervention or reoperation, with increased morbidity and mortality. Because conventional bypass grafting has traditionally used a combination of SVGs and internal mammary arteries, late vein graft failure results in a significant impact on the late results after coronary bypass grafting. 5

When harvesting vein grafts for coronary bypass, there is an ischemic interval of 30 to 60 minutes, during which the vein is usually kept in a balanced salt preservative solution. The early events of vein graft preparation and their link to intimal hyperplasia have been the focus of a number of studies ${ }^{6,7}$ but, despite a great number of experimental studies, the mechanisms regulating the vein graft response to injury during preparation and arterialization remain elusive.

In arterial vascular injury, the mitogen-activated protein kinase (MAPK) pathways strongly influence vascular smooth muscle cell proliferation, migration, and apoptosis, ${ }^{8,9}$ key factors in the pathogenesis neointimal formation and late restenosis. Similarly, results of both in vivo and in vitro studies suggest a role for MAPKs in the venous response to injury. ${ }^{10,11}$ In this study, we investigated the MAPK pathways in SVGs at the time of initial harvesting and at the end of incubation in balanced salt solution, immediately prior to surgical implantation, to determine whether these manipulations resulted in activation of the MAPK pathways. In addition, the time sequence of MAPK activation was tested in a canine model.

\section{Methods}

Human saphenous vein segments from 10 patients were excised and processed for use as conduits for coronary artery bypass procedures. Approval by the institutional review board as well as informed consent were obtained from each patient. Periadventitial tissue was dissected and the vein distended by handheld injection of saline solution. As soon as the saphenous vein harvest was completed, the distal $3 \mathrm{~cm}$ were passed off the operative field and transected into 3 equal segments using a sterile blade. One segment was processed as a control at $\mathrm{t}=0$ by immediately placing it into ice-cold lysis buffer $(50 \mathrm{mmol} / \mathrm{L}$ of 6-[2-hydroxyethyl]-1-piperazineethanesulfonic, $\mathrm{pH} 7.5,150 \mathrm{mmol} / \mathrm{L}$ of $\mathrm{NaCl}, 1 \mathrm{mmol} / \mathrm{L}$ of ethylenediaminetetraacetic acid, $10 \%$ glycerol, $1 \%$ Triton-X-100, $25 \mathrm{mmol} / \mathrm{L}$ of sodium fluoride) containing protease inhibitor cocktail (Roche, Indianapolis, Ind) and $1 \mathrm{mmol} / \mathrm{L}$ of sodium orthovanadate (Sigma, St Louis, Mo). The remaining 2 segments were left at room temperature in normal saline until the proximal anastomosis of the aortocoronary vein grafts was started. In some cases either PD98059 (50 mmol/L) $(\mathrm{n}=3)$, an inhibitor of extracellular regulated kinase (ERK) activation, or $0.1 \%$ dimethylsulfoxide (DMSO; vehicle) $(\mathrm{n}=3)$ was added to the saline solution. The mean time from harvesting of the vein conduit until performance of the proximal anastomoses $(t=1)$ was 50 minutes. At $t=1$, the remaining vein specimens were removed from their incubation solution and placed into ice-cold lysis buffer. All vein segments were each finely minced and homogenized by sonication (Branson Sonifier 450; VWR Scientific Products, Bridgeport, NJ) and the homogenates clarified by centrifugation at 14,000 rpm for $5 \mathrm{~min}$ utes in a refrigerated Eppendorf centrifuge. The supernatant was removed and stored at $-80^{\circ} \mathrm{C}$ for future use.

To perform kinetics and characterization studies of MAPK activation on a more controllable model, saphenous veins were also harvested from adult male mongrel dogs $(25 \mathrm{~kg})$. All animals have received humane care in compliance with the Guide for the Care and Use of Laboratory Animals prepared by the Institute of Laboratory Animal Resources, National Research Council. The canine saphenous veins were harvested as described above, with the exception that in most experiments phosphate-buffered saline $(\mathrm{pH}$ 7.4) was used instead of simple saline solution ( $\mathrm{pH} 5.9)$, mostly because preliminary experiments showed no difference on MAPK activation between the 2 solutions. Each saphenous vein was divided into 5 equal segments. The vein samples were harvested after $0,5,15,30$, and 60 minutes and processed as described above, except that the canine vein segments were not treated with either PD98059 or DMSO.

\section{Western Blotting}

Equal amounts of protein from each vein sample were loaded onto sodium dodecylsulfate-10\% polyacrylamide gels and electrophoresed. After separation, proteins were transferred onto a polyvy- 


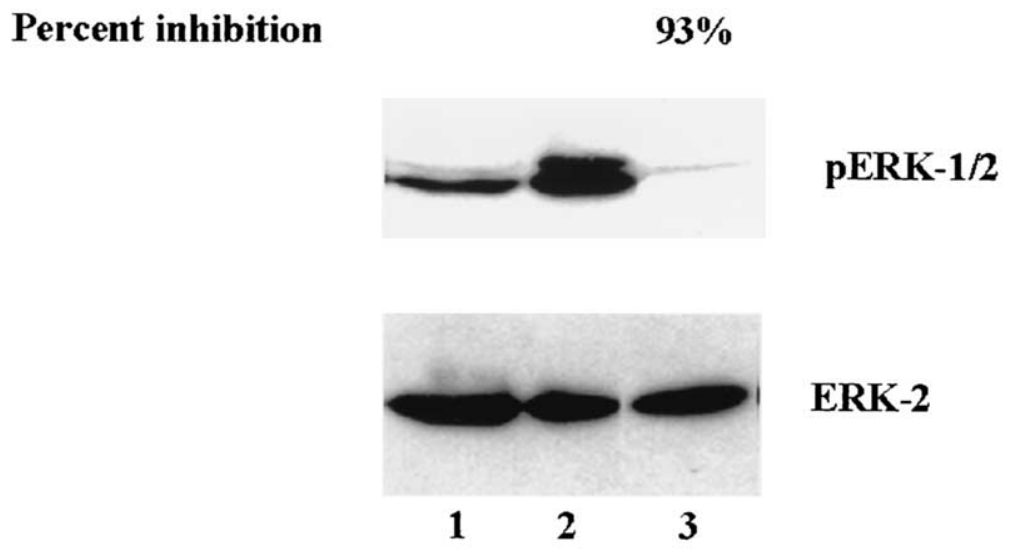

Figure 1. ERK activation in human saphenous vein being prepared for bypass surgery. Saphenous vein segments were prepared and treated as described in Materials and Methods and analyzed for activation of the ERK pathway. Upper panel: Western blotting analysis with anti-phospho-ERK (p-ERK-1/2) antibody. Lane 1, control, time 0; lane 2, 50-minute incubation in normal saline; lane 3,50-minute incubation in normal saline with PD98059, a specific inhibitor of ERK activation. Lower panel: The same membrane was stripped and incubated with an anti-ERK-2 (ERK-2) antibody to control for equal transfer and loading. Quantitation of ERK-1/2 activation was performed as described in Materials and Methods. Inhibition of ERK-1/2 activation achieved with PD98059 (lane 3) is indicated as a percentage as compared with activation at $t=1$ (lane 2) (top of the upper panel).

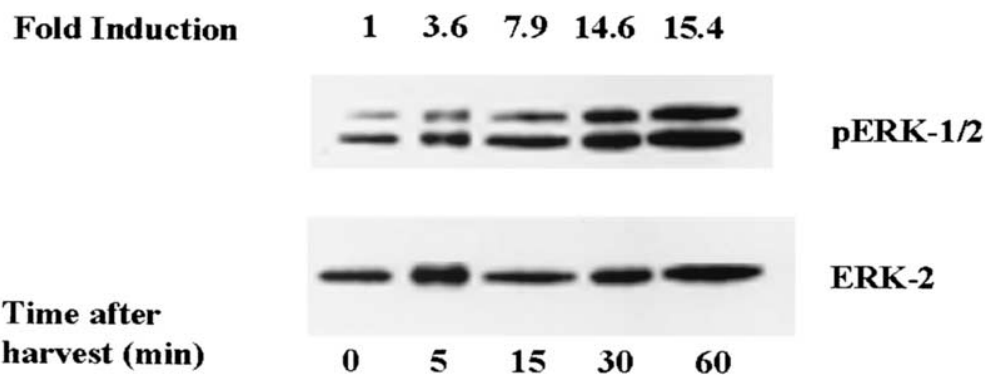

Figure 2. ERK activation in canine saphenous vein. Western blotting analysis of ERK-1/2 activation in canine saphenous vein at time of harvest $(0)$ and after incubation in normal saline at indicated time points. Upper panel: Western blotting with anti-active ERK antibody (pERK-1/2). Lower panel: The same membrane was stripped and incubated with anti-ERK-2 (ERK-2) antibody to control for equal loading and transfer. Quantitation of ERK-1/2 activation was performed as outlined in Materials and Methods and expressed as fold induction over the control $(\mathbf{t}=\mathbf{0})$ (top of upper panel).

nilidene fluoride membrane (Immobilon P; Millipore, Bedford, Mass) and the membranes were blocked with Tris-buffered saline (TBS) containing 5\% bovine serum albumin for 2 hours at room temperature. Membranes were then incubated overnight with the following antibodies: (1) anti-active ERK polyclonal antibody (Cell Signaling Technology, Beverly, Mass) followed, after stripping of the same membrane, by anti-total ERK-2 polyclonal antibody (Santa Cruz Biotechnology Inc, Santa Cruz, Calif); and (2) anti-active Akt polyclonal antibody (Cell Signaling Technology) followed, after stripping of the same membrane, by anti-total Akt polyclonal antibody (Cell Signaling Technology). When the incubation with the first antibody was completed, membranes were washed with TBS containing $0.05 \%$ Tween 20 , and then with TBS alone, and subsequently incubated for 1 hour with horseradish peroxidase-linked anti-rabbit immunoglobulin $\mathrm{G}$ antibody. After thorough washes of the membrane, protein bands were detected by enhanced chemiluminescence (Roche) and analyzed using Kodak 1D Image software (Kodak, Rochester, NY).

Initial analysis of c-jun N-terminal kinase (JNK) activation was performed using an anti-active JNK antibody (Cell Signaling Technology) for Western blotting. This method revealed inconsistent data. Therefore, JNK activity was analyzed using an in vitro kinase assay. This was performed by using a glutathione $S$-transferase-Jun fusion protein to affinity-purify JNK and as its substrate as well (Cell Signaling Technology). Subsequently, glutathione $S$-transferase-Jun phosphorylation was detected by Western blotting using an anti-phospho-c-Jun polyclonal antibody (Cell Signaling Technology) followed, after stripping of the same mem- 


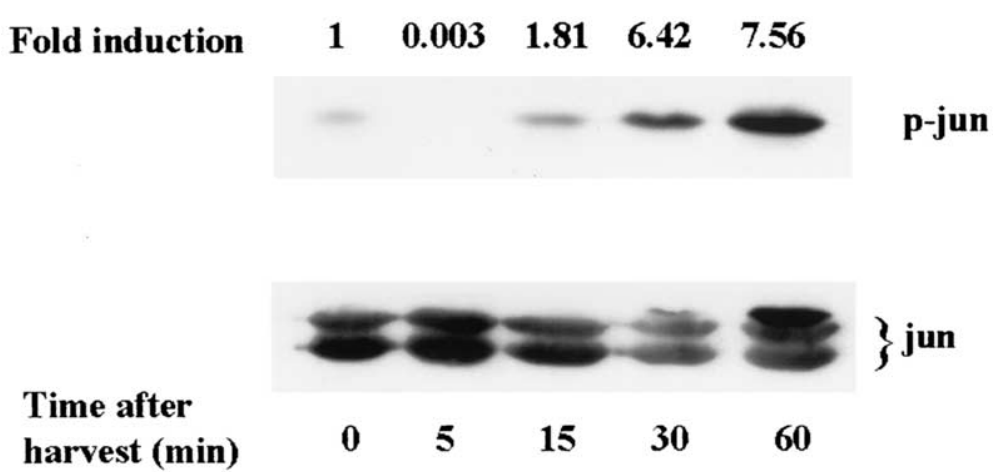

Figure 3. JNK activation in canine saphenous vein. In vitro kinase assay of JNK activation in canine saphenous vein at time of harvest $(0)$ and after incubation in normal saline at indicated time points was performed by analyzing phosphorylation of a GST-jun fusion protein as described in Materials and Methods. Upper panel: Analysis of the phosphorylated form of GST-jun with an anti-phospho-c-jun (p-jun) antibody. Lower panel: The same membrane was stripped and incubated with an anti-c-jun (jun) antibody to control for equal loading and transfer. Two bands are recognized as the GST-jun preparation contains 2 different products (only the higher molecular weight GST-jun undergoes phosphorylation). Quantitation of JNK activation was performed as outlined in Materials and Methods and expressed as fold induction over the control $(\mathrm{t}=0)$ (top of upper panel).

\section{$\begin{array}{llllll}\text { Fold induction } & 1.0 & 0.89 & 1.02 & 3.37 & 3.82\end{array}$}

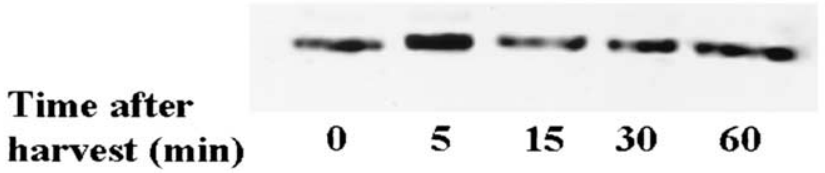

Akt

Figure 4. Akt activation in canine saphenous vein. Western blotting analysis of Akt activation in canine saphenous vein at time of harvest $(0)$ and after incubation in normal saline at indicated time points. Upper panel: Western blotting with anti-phospho-Akt ( $p$-Akt) antibody. Lower panel: The same membrane was stripped and incubated with anti-total Akt (Akt) antibody to control for equal loading and transfer. Quantitation of Akt activation was performed as outlined in Materials and Methods and expressed as fold induction over the control $(t=0)($ top of upper panel).

brane, by incubation with anti-total c-Jun polyclonal antibody (Cell Signaling Technology). Proteins were then detected and analyzed as described above.

\section{Results}

Human saphenous vein samples were analyzed for ERK activation as described above. At the end of the incubation period $(\mathrm{t}=1$; mean $=50$ minutes $)$, ERK activation was found to be increased approximately twofold as compared with the vein graft immediately after excision $(\mathrm{t}=0)$. Incubation with the mitogen-activated protein kinase kinase (MEK)-1/2 inhibitor PD98059 resulted in a strong inhibition $(>90 \%)$ of ERK activation (Figure 1).
The time course of ERK and JNK activation was then studied in more detail in saphenous vein specimens excised from dogs. Figure 2 shows that ERK activation increased over a period of 60 minutes, peaking at 30 minutes after vein excision. Analysis of JNK activation showed similar kinetics of activation (Figure 3), suggesting that both ERK and JNK pathways might be activated by the same factor(s). A variety of stimuli may be responsible for the activation of these MAPKs. These likely include growth factors that are released during excision and/or manipulation of vein grafts. To test this hypothesis, we analyzed the PI3 kinase pathway because this pathway is also activated upon growth factors binding to their specific receptor tyrosine kinases. ${ }^{12}$ Figure 


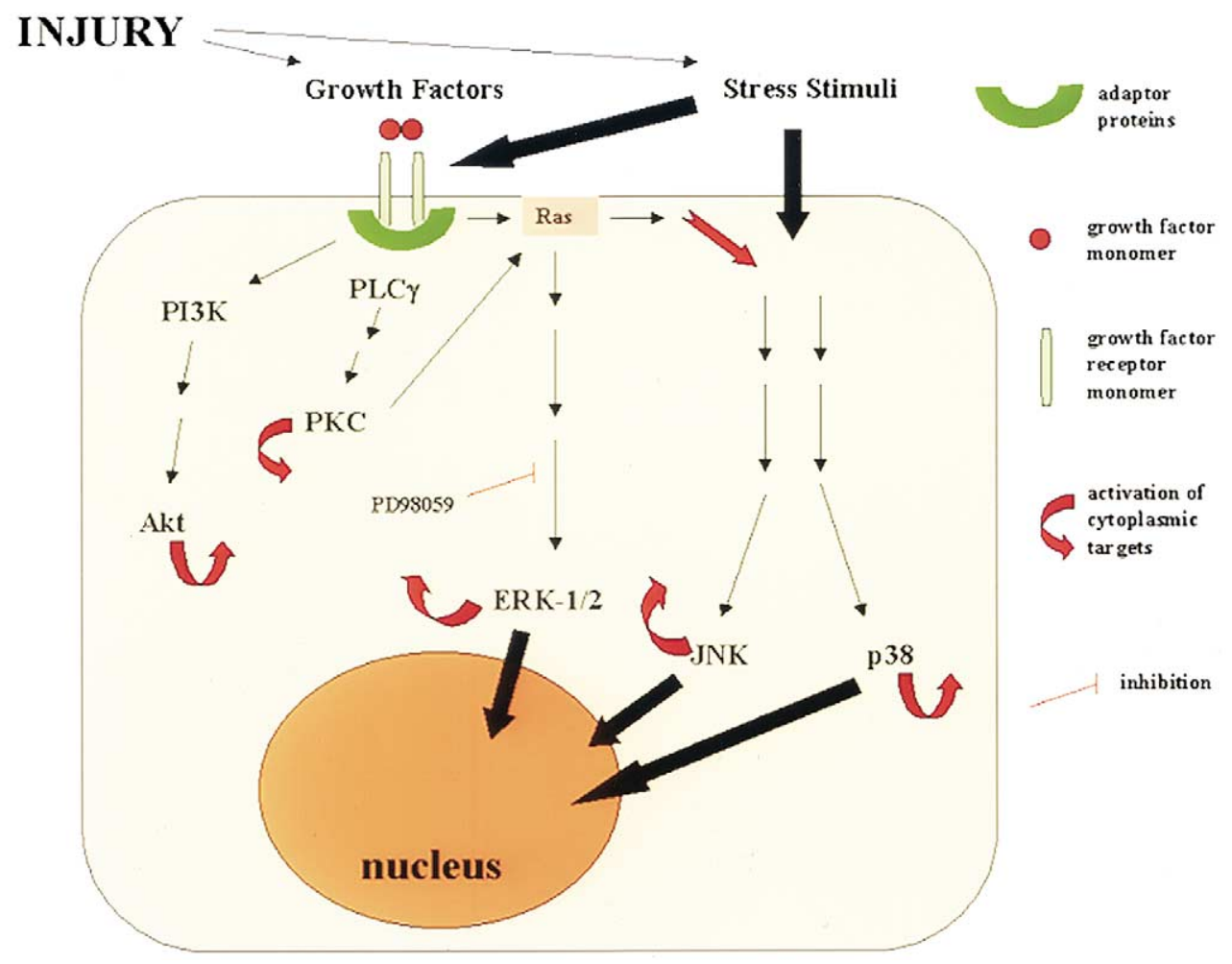

Figure 5. Proposed mechanism of intracellular signaling upon injury due to vein graft preparation. Upon injury, growth factors released from neighboring cells and extracellular components activate their specific receptor tyrosine kinases. Dimerization and cross-phosphorylation of growth factor receptors prompt adaptor proteins to initiate a series of cascades, including: (1) activation of the PI3 kinase (PI3K) pathway, and consequent Akt (also known as protein kinase B) phosphorylation; (2) activation of phospholipase C-gamma (PLC $\gamma$ ) and consequent protein kinase $C$ (PKC) activation; (3) recruitment of the small guanosine 5 'triphosphate (GTP)-binding protein Ras to the cell membrane and consequent activation of the ERK-1/2 pathway. On the other hand, vein preparation also involves stretching, oxidative, and inflammatory cytokine stress, schematically grouped here as stress stimuli. Cells sense these stimuli both by growth factor receptor clustering and stimulation, which can also result in involvement of additional GTP-binding proteins leading to stress pathway activation, and/or by directly activating specific stress pathways culminating into activation of the stress-activated MAPKs JNK and p38. MAPKs (ERK, JNK, and p38) are, therefore, the ultimate acceptors of phosphate groups between the stimulus at the cell membrane and the nucleus. Upon activation, MAPKs can activate nuclear proteins (generally transcription factors), which results in transcriptional activation of discrete chromosomal regions, and/or activate specific cytoplasmic targets. The duration and integration of these activated pathways will determine profound phenotypic modifications such as cell proliferation, migration, programmed death (apoptosis), and metabolic changes. Please note that the schematic also shows that Akt and PKC activate specific protein targets and that a cross talk between PKC and ERK-1/2 activation has been demonstrated in many systems, further strengthening the central role of MAPKs as ultimate effectors. Thin arrows indicate the multistep nature of each pathway. The role of PD98059 as an upstream inhibitor of ERK-1/2 activation is also indicated.

4 shows that the PI3 kinase pathway, studied by monitoring activation of Akt, a downstream target, demonstrated the same kinetics observed for ERK and JNK activation.

\section{Discussion}

MAPKs have been identified as key intracellular signaling mediators of cellular phenotypic changes. ${ }^{9}$ Three major MAPK pathways have been characterized: (1) extracellular signal-regulated kinases (ERKs), (2) stress-activated protein kinases (that are responsible for the phosphorylation of the transcription factor c-jun and therefore referred to as SAPKs or JNKs), and (3) stress-activated $\mathrm{p} 38^{\mathrm{MAPK}}$. It is generally assumed that these pathways, initiated at the cell surface, ultimately lead to phosphorylation of cytoplasmic as well as nuclear targets, including a number of transcription factors, which determines a change of transcriptional activity. The 
ERK pathway is predominantly activated following the interaction of growth factors with their specific surface tyrosine kinase receptors; in contrast, JNK and $\mathrm{p} 38^{\mathrm{MAPK}}$ are highly responsive to stress stimuli. ${ }^{9}$ These distinctions are not absolute, however. In fact, it is the integration among different intracellular signaling pathways and their kinetics of activation that determines cellular phenotypes such as cell proliferation, migration, differentiation, and apoptosis. ${ }^{9,13,14}$

As noted above, ERKs are most efficiently activated by growth factors. One key growth factor and potent angiogenesis inducer, basic fibroblast growth factor (FGF-2), that has been implicated in the vascular response to injury and in the development of intimal hyperplasia ${ }^{15,16}$ appears to work at least in part via the ERK pathway. In cultured endothelial cells, we have previously shown that FGF-2 controls cell migration secondary to mechanical wounding through activation of the ERK pathway. ${ }^{17}$ FGF-2 is also the key regulator of this response, as its deficiency alone impairs both ERK activation and endothelial cell wound repair. ${ }^{18}$ It is possible, therefore, that FGF-2 and/or other growth factors control the early vascular response in vein grafts by activating specific intracellular pathways, including the MAPKs. This possibility is strongly supported by the observation that in excised saphenous veins Akt phosphorylation, and therefore activation, follows the same kinetics of MAPK activation (Figure 4). Because the growth factorinitiated PI3 kinase pathway tightly regulates Akt phosphorylation, this result reinforces the hypothesis that activation of these intracellular signaling pathways involving the MAPKs occurs via growth factor receptor(s). On the other hand, mechanical injury and stretching have been implicated in MAPK activation; therefore, it is likely that the intracellular pathways investigated here are activated by multiple etiological factors (Figure 5).

Veins are still the most commonly used conduits for arterial bypass surgery. ${ }^{5}$ Vein remodeling secondary to the injuries associated with arterialization is characterized by damage to and loss of endothelium, together with smooth muscle cell proliferation and migration. This process is also characterized by abundant deposition of extracellular matrix in the expanded neointima. ${ }^{19-21}$ Although the molecular mechanism(s) that control intimal hyperplasia in vein grafts have not been fully elucidated, numerous studies on cultured vascular endothelial and smooth muscle cells or on in vivo injured arteries have unequivocally linked MAPK activation to cell proliferation, migration, and apoptosis. ${ }^{17,22-24}$ To date no studies have examined MAPK activation in vein grafts, however, even though vein grafts are ideal candidates for ex vivo molecular treatments, ${ }^{25}$ entailing minimal or no systemic effects.

The results of the present study demonstrate that saphenous vein excision and preparation result in activation of both ERKs and JNKs. The increase in ERK activity is sustained but can be pharmacologically reduced by simply incubating the whole vessel in a solution containing a specific MEK-1/2 inhibitor. The results reported here are consistent with reports of the activation of ERK-1/2 in arteries following either balloon injury or fluid shear stress. ${ }^{8,26,27}$ Furthermore, the present results are consistent with the ERK activation observed after mechanical injury of cultured endothelial cells. ${ }^{17,18}$ Effective inhibition of ERK by synthetic inhibitors has very significant implications. In fact, in cultured endothelial cells inhibition of the ERK pathway completely suppresses cell migration, ${ }^{17,18}$ although in a balloon injury model, ERK inhibition decreases the amount of medial smooth muscle cell replication, a key process in the development of intimal hyperplasia. ${ }^{8}$ Similar to what has been described in arterial injury, JNK activation levels were also found to increase after vein graft harvesting.

Significantly, this study represents the first attempt to characterize activation and kinetics of MAPKs in both human and canine veins being prepared for bypass grafting. Numerous studies have pointed out the importance of mechanical trauma that occurs during pregrafting manipulation of vein conduits. ${ }^{6,7,28}$ Within this context, the finding that both ERK and JNK pathways are up-regulated after vein harvesting may have significant impact in understanding the mechanisms that lead to intimal hyperplasia and late graft failure. However, the results shown in this report cannot clarify the role(s) (if any) of MAPK activation in determining the cellular changes that lead to intimal hyperplasia of grafted veins. In fact, one should keep in mind that, following excision and preparation, a vein graft undergoes a series of additional stresses, including mechanical and shear stress due to integration into the arterial flow, as well as inflammatory reactions brought to the vessel wall both by circulating cells and/or cytokines. For these reasons, the inherent intracellular response(s) cannot be identified within the system described in the present report. Further studies are underway in our laboratory to determine the influence of vein graft arterialization on MAPK activation and, most importantly, the relevance of these pathways in modulating the development of intimal hyperplasia and graft durability.

\section{References}

1. Austin GE, Ratliff NB, Hollman J, Tabei S, Phillips DF. Intimal proliferation of smooth muscle cells as an explanation for recurrent coronary artery stenosis after percutaneous transluminal coronary angioplasty. J Am Coll Cardiol. 1985;6:369-75.

2. Sayers RD, Watt PA, Muller S, Bell PR, Thurston H. Structural and functional smooth muscle injury after surgical preparation of reversed and non-reversed (in situ) saphenous vein bypass grafts. Br J Surg. 1991;78:1256-8.

3. Bryan AJ, Angelini GD. The biology of saphenous vein graft occlusion: etiology and strategies for prevention. Curr Opin Cardiol. 1994; 9:641-9.

4. Barner HB, Standeven JW, Reese J. Twelve-year experience with 
internal mammary artery for coronary artery bypass. $J$ Thorac Cardiovasc Surg. 1985;90:668-75.

5. Thatte HS, Khuri SF. The coronary artery bypass conduit: I. Intraoperative endothelial injury and its implication on graft patency. Ann Thorac Surg.. 2001;72:S2245-52 (discussion S2267-70).

6. Baumann FG, Catinella FP, Cunningham JN Jr, Spencer FC. Vein contraction and smooth muscle cell extensions as causes of endothelial damage during graft preparation. Ann Surg. 1981;194:199-211.

7. Catinella FP, Cunningham JN Jr, Srungaram RK, et al. The factors influencing early patency of coronary artery bypass vein grafts: correlation of angiographic and ultrastructural findings. $J$ Thorac Cardiovasc Surg. 1982;83:686-700.

8. Koyama H, Olson NE, Dastvan FF, Reidy MA. Cell replication in the arterial wall: activation of signaling pathway following in vivo injury. Circ Res. 1998;82:713-21.

9. Bogoyevitch MA. Signalling via stress-activated mitogen-activated protein kinases in the cardiovascular system. Cardiovasc Res. 2000; 45:826-42.

10. Mayr M, Li C, Zou Y, Huemer U, Hu Y, Xu Q. Biomechanical stress-induced apoptosis in vein grafts involves p38 mitogen-activated protein kinases. Faseb J. 2000;14:261-70.

11. Xu Q. Biomechanical-stress-induced signaling and gene expression in the development of arteriosclerosis. Trends Cardiovasc Med. 2000;10: $35-41$.

12. Schlessinger J. Cell signaling by receptor tyrosine kinases. Cell. 2000; 103:211-25.

13. Cowley S, Paterson H, Kemp P, Marshall CJ. Activation of MAP kinase kinase is necessary and sufficient for PC12 differentiation and for transformation of NIH 3T3 cells. Cell. 1994;77:841-52.

14. Marshall CJ. Specificity of receptor tyrosine kinase signaling: transient versus sustained extracellular signal-regulated kinase activation. Cell. 1995;80:179-85.

15. Lindner V, Reidy MA. Proliferation of smooth muscle cells after vascular injury is inhibited by an antibody against basic fibroblast growth factor. Proc Natl Acad Sci U S A. 1991;88:3739-43.

16. Nguyen HC, Steinberg BM, LeBoutillier M 3rd, et al. Suppression of neointimal lesions after vascular injury: a role for polyclonal anti-basic fibroblast growth factor antibody. Surgery. 1994;116:456-61 (discussion 461-2).
17. Pintucci G, Steinberg BM, Seghezzi G, et al. Mechanical endothelial damage results in basic fibroblast growth factor-mediated activation of extracellular signal-regulated kinases. Surgery. 1999;126:422-7.

18. Pintucci G, Moscatelli D, Saponara F, et al. Lack of ERK activation and cell migration in FGF-2-deficient endothelial cells. Faseb J. 2002; 16:598-600

19. Schwartz SM, Reidy MA, O'Brien ER. Assessment of factors important in atherosclerotic occlusion and restenosis. Thromb Haemost. 1995;74:541-51.

20. Riessen R, Wight TN, Pastore C, Henley C, Isner JM. Distribution of hyaluronan during extracellular matrix remodeling in human restenotic arteries and balloon-injured rat carotid arteries. Circulation. 1996;93: 1141-7.

21. Newby AC. An overview of the vascular response to injury: a tribute to the late Russell Ross. Toxicol Lett. 2000;112-113:519-29.

22. Bornfeldt KE, Campbell JS, Koyama H, et al. The mitogen-activated protein kinase pathway can mediate growth inhibition and proliferation in smooth muscle cells. Dependence on the availability of downstream targets. J Clin Invest. 1997;100:875-85.

23. Lille S, Daum G, Clowes MM, Clowes AW. The regulation of p42/p44 mitogen-activated protein kinases in the injured rat carotid artery. J Surg Res. 1997;70:178-86.

24. Boilly B, Vercoutter-Edouart AS, Hondermarck H, Nurcombe V, Le Bourhis X. FGF signals for cell proliferation and migration through different pathways. Cytokine Growth Factor Rev. 2000;11:295-302.

25. Fernandez HA, Kallenbach K, Seghezzi G, et al. Inhibition of endothelial cell migration by gene transfer of tissue inhibitor of metalloproteinases-1. J Surg Res. 1999;82:156-62.

26. Pyles JM, March KL, Franklin M, Mehdi K, Wilensky RL, Adam LP. Activation of MAP kinase in vivo follows balloon overstretch injury of porcine coronary and carotid arteries. Circ Res. 1997;81:904-10.

27. Li S, Kim M, Hu YL, et al. Fluid shear stress activation of focal adhesion kinase. Linking to mitogen-activated protein kinases. $J$ Biol Chem. 1997;272:30455-62.

28. Engstrom KG, Szentkiralyi I. Impaired contractility of left-over vein grafts used for $\mathrm{CABG}$ and the possible trauma caused by air exposure. Scand Cardiovasc J. 2001;35:403-8. 